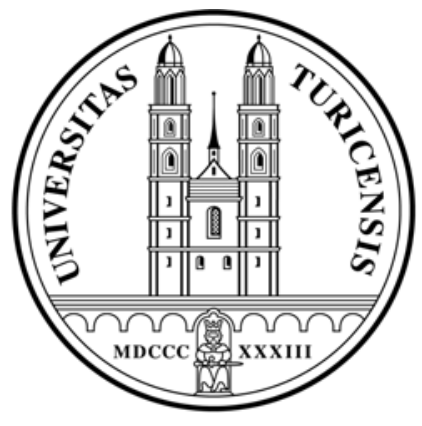

Institute for Empirical Research in Economics

University of Zurich

Working Paper Series

ISSN 1424-0459

Working Paper No. 422

\title{
Lottery pricing under time pressure
}

Pavlo R. Blavatskyy and Wolfgang R. Köhler

July 2009 


\title{
Lottery pricing under time pressure
}

\author{
Pavlo R. Blavatskyy ${ }^{*}$ and Wolfgang R. Köhler ${ }^{\dagger}$
}

March 2009

\begin{abstract}
This paper investigates how subjects determine minimum selling prices for lotteries. We design an experiment where subjects have at every moment an incentive to state their minimum selling price and to adjust the price if they believe that the price that they stated initially was not optimal. We observe frequent and sizeable price adjustments. We find that random pricing models can not explain the observed price patterns. We show that earlier prices contain information about future price adjustments. We propose a model of Stochastic Pricing that offers an intuitive explanation for these price adjustment patterns.
\end{abstract}

Key words: time pressure, certainty equivalent, experiment, stochastic, Becker-DeGrootMarschak (BDM) method

JEL Classification codes: C91, D81

\footnotetext{
* Corresponding author, Institute for Empirical Research in Economics, University of Zurich, Winterthurerstrasse 30, CH-8006 Zurich, Switzerland, tel.: +41(0)446343586, fax: +41(0)446344978, e-mail: pavlo.blavatskyy@iew.unizh.ch

${ }^{\dagger}$ Institute for Empirical Research in Economics, University of Zurich, Winterthurerstrasse 30, CH-8006 Zurich, Switzerland, tel.: +41(0)446343588, fax: +41(0)446344978, e-mail: wolfgang.koehler@iew.unizh.ch
} 


\section{Introduction}

This paper investigates how individuals determine minimum selling prices for lotteries. In a standard lottery pricing experiments, we observe one price for each lottery. ${ }^{1}$ The price that is observed by the experimenter is the one that the subject enters after some time of deliberation. This paper tries to shed some light on how does this deliberation process works and on how individuals determine minimum selling prices.

We ran an experiment where 60 subjects had to price various lotteries. The objective of the experiment was to observe the deliberation process, i.e., to observe how the minimum selling price evolves/emerges over time. Therefore, we designed the experiment such that subjects had during a pricing task at every moment an incentive to state their minimum selling price and to adjust the price if they believe that the price that they stated initially was not optimal.

To achieve this, we use the following experimental design: The computer screen displayed the lottery and a list of prices spaced equally apart between zero and the highest outcome of the lottery (see screenshot in the appendix). Subjects were asked to indicate their minimum selling price by clicking on one of the prices on the list. Subjects were told that the question is terminated after some randomly determined time (between 10 and 30 seconds) and that the last price is used to determine the payoffs. If subjects did not click on a price before the question was terminated, they received a payoff of zero if the question was selected to determine their payoffs. Subjects could adjust the price as often as they wanted. The computer program recorded the order in which prices are clicked on. The last price that a subject clicked on was used to determine her payoff by the standard Becker-

\footnotetext{
${ }^{1}$ A large number of experiments elicit certainty equivalents via a sequence of binary choices (e.g. Tversky and Kahneman 1992). In the context of this paper, we do not consider these experiments as pricing tasks, since subjects do not state prices, Instead, certainty equivalents are inferred from the observed binary choices. These experiments ask subjects to make several binary choices but not to price lotteries.
} 
DeGroot-Marschak-procedure (Becker et al. 1964). Hence, subjects had an incentive to state minimum selling price at any time (except for first ten seconds).

We observed that in $78 \%$ of the tasks, subjects adjusted their price at least once, in $53 \%$ of the tasks, subjects adjusted the price at least twice. Subjects did not only adjust prices frequently, they also made quite substantial adjustments within a short period of time. In $35 \%$ of all cases, subjects adjusted their prices by more than $10 \%$ of the highest outcome of the lottery. We interpret our results as evidence that decision making takes time and that subjects gradually refine their decision/answer over time until they arrive after sufficient deliberation at a final price.

The vast majority of decision theories is concerned with explaining and predicting prices for lotteries but does not discuss how these prices are determined (a notable exception is the computational model of Johnson and Busemeyer (2005)). Since we observe that subjects adjust their price, the question arises whether these price adjustments are random or whether the observed price adjustment patterns allow to draw conclusions on how subjects determine the minimum selling price of a lottery. We find that random pricing models where subjects receive iid. draws from some distribution of prices can not explain the price patterns that we observe in the experiment. Instead, we show that earlier prices contain information about future price adjustments.

Blavatskyy and Köhler (2009) propose a model of how individuals price lotteries that models explicitly the process that subjects use to determine minimum selling prices. The model considers subjects whose preferences are described by a random utility model. Subjects determine the minimum selling price of a lottery via a sequence of hypothetical binary comparisons between the lottery and different monetary amounts. Depending on whether the amount or the lottery is preferred, the subject decreases or increases the amount to which the lottery is compared. 
This model of Stochastic Pricing can explain the price adjustment patterns that we observe. We run a Monte-Carlo simulation to compare the predictions of the model with the experimental results. The model predicts frequent and sizeable price adjustments. The pricing patterns that are generated by the model match the patterns that we observe in the experiment. Additionally, the model offers a simple explanation for why the price adjustment patterns differ for lotteries that yield the high outcome with small and large probability.

To achieve our objective that subjects had at every moment an incentive to state their minimum selling price we used a form of 'continuous' time pressure. Since subjects did not know when the task was terminated, they had an incentive to state a price quickly and adjust the price if they believed that the price that they stated earlier was not optimal. While these procedure is related to experiments that analyse decision making under time pressure (e.g. Wright, 1974; Ben Zur and Breznitz, 1981; Busemeyer, 1985, 1993), it is not our primary aim to analyse how time pressure affects decisions. Instead, we use time pressure only as a tool to observe how the minimum selling price evolves/emerges over time.

The remainder of this paper is organized as follows. Section 2 describes the design and implementation of the experiment. Section 3 presents the experimental results. Section 4 shows that random pricing models cannot accommodate the experimental results. Section 5 shows that the patterns of price adjustments observed in the experiment are consistent with a simple procedural model of stochastic pricing, where individuals search for their minimum selling price through a sequence of binary comparisons. Section 6 concludes. 


\section{Experimental Design and Implementation}

We used the 6 lotteries that are shown in Table 1. All lotteries have only two outcomes and the lowest outcome is zero. Outcomes are in Swiss Francs. At the time of the experiment, one Swiss Franc was approximately $\$ 0.83$ or $€ 0.61$. Lotteries were described and subsequently played out in terms of the number of red and black cards in a box that contains 100 cards. The computer screen displayed the lottery and a list of prices spaced equally apart between zero and the highest outcome of the lottery (see screenshot in the appendix). The number of prices was chosen such that all prices on the list are integers.

\begin{tabular}{|c|c|c|c|c|c|c|}
\hline Lottery & L1 & L2 & L3 & L4 & L5 & L6 \\
\hline Outcome $x_{1}$ & 150 & 120 & 90 & 96 & 60 & 84 \\
\hline Probability $p_{1}$ & 0.15 & 0.22 & 0.35 & 0.45 & 0.68 & 0.85 \\
\hline Outcome $x_{2}$ & 0 & 0 & 0 & 0 & 0 & 0 \\
\hline Probability $p_{2}$ & 0.85 & 0.78 & 0.65 & 0.55 & 0.32 & 0.15 \\
\hline \# of prices on list & 31 & 31 & 31 & 33 & 31 & 29 \\
\hline
\end{tabular}

Table 1 Lotteries (Outcomes are in Swiss Francs)

The objective of the experiment was to observe how the minimum selling price evolves over time. Therefore, we designed the experiment such that subjects had at every moment an incentive to state their minimum selling price and to adjust the price if they believe that the price that they stated initially was not optimal.

Subjects were asked to indicate their minimum selling price by clicking on one of the prices on the list. Subjects were told that the question is terminated after some randomly determined time (between 10 and 30 seconds) and that the last price is used to determine the payoffs. If subjects did not click on a price before the question was terminated, they received a payoff of zero if the question was selected to determine their 
payoffs. Subjects could adjust the price as often as they wanted. The computer program recorded the order in which prices are clicked on. The last price that a subject clicked on was used to determine her payoff by the standard Becker-DeGroot-Marschak-procedure.

If one of these tasks was selected to determine the earnings, a random price was drawn from the interval between zero and the highest outcome of the lottery. If the last price that the subject entered was higher than the price that was drawn, she would play the lottery. Otherwise she would sell the lottery and receive the price that was drawn.

When subjects had to play one of these tasks, they first received a notification. The notification told them that they would receive a payoff of zero if they did not enter a price before the task was terminated. After the notification, one of the lotteries and the corresponding list of prices appeared on the screen and time started running.

\subsection{Implementation of the experiment}

The experiment was conducted in the experimental laboratory of the Institute for Empirical Research in Economics at the University of Zürich. Sixty undergraduates (35 male and 25 female) from a variety of majors participated in the experiment. The average age was 22. There were two sessions with 30 subjects in each session. At the beginning of the experiment, subjects received a copy of the instructions (a translation can be found in the Appendix). Instructions included screenshots for the different tasks. Additionally, the experimenter read aloud the instructions. The experiment lasted about 45 minutes (plus 30 minutes to explain the instructions). The experiment was programmed with z-Tree (Fischbacher, 2007). The six pricing tasks that are analyzed in this paper were presented to subjects in a random order intermixed with 66 other decision problems that will be analyzed elsewhere (cf. Blavatskyy and Köhler, 2009).

We used a random lottery incentive scheme and physical randomization devices. At the end of the experiment each subject drew a card from a box with cards numbered 
from 1 to 72 (total number of decision problems). The number on the card determined the decision problem which was used to compute the payoff of the subject. If the subject had to play a risky lottery, she had to draw a second card from a box with the corresponding distribution of red and black cards (we used standard playing cards). Subjects drew the second card outside the main laboratory to preserve the anonymity of payments.

Subjects received a $10 \mathrm{CHF}$ show-up fee and whatever they earned in the experiment. Average earnings were 43.9 CHF (approx. \$40 or €27). The lowest earning was $10 \mathrm{CHF}$, the highest was $133.8 \mathrm{CHF}$. At the end of the experiment, the subjects were asked to complete a short socio-demographic questionnaire.

\section{Results}

We first present some descriptive statistics which summarize the results of the experiment. Figure 1 presents the distribution of the number of price adjustments that subjects made in the experiment (across 6 lotteries and 60 subjects). In $78 \%$ of the tasks, subjects adjusted their price at least once, in 53\% of the tasks, subjects adjusted the price at least twice.

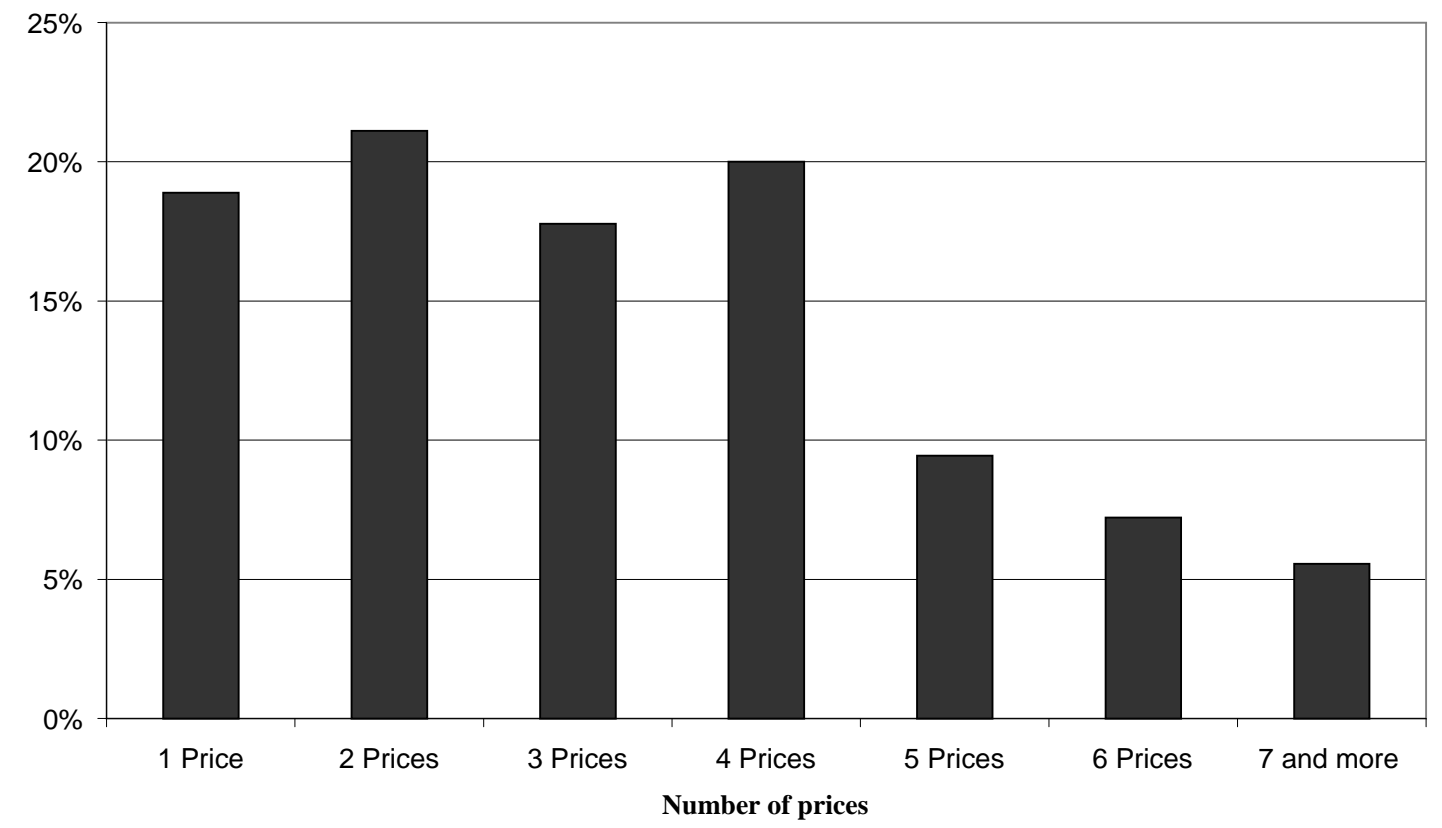

Figure 1 Percentage of subjects stating as many prices as indicated on the horizontal axis 
Subjects could adjust their price at zero cost. The last price that was entered was used to determine the payoffs. Since the tasks were terminated at some randomly selected time, subjects had an incentive to state the best estimate of their minimum selling price at every point in time. ${ }^{2}$ In all tasks, subjects stated at least one price before the task was terminated. Table 2 shows for each lottery the time limit and the average number of prices that subjects state. Subjects adjust their price more frequently if they have more time to do so. This indicates that the determination of the minimum selling price takes some time.

\begin{tabular}{|c|c|c|c|c|c|c|}
\hline Lottery & L1 & L2 & L3 & L4 & L5 & L6 \\
\hline Time Limit (sec.) & 19 & 27 & 17 & 22 & 21 & 18 \\
\hline $\begin{array}{c}\text { Average \# of prices } \\
\text { per subject }\end{array}$ & 2.87 & 3.9 & 3 & 3.2 & 3 & 2.6 \\
\hline
\end{tabular}

Table 2 Average number of stated prices

Subjects did not only adjust prices frequently, they also made quite substantial adjustments within a short period of time. Figure 2 shows the distribution of the discrepancy between the maximum and the minimum price that a subject states for a lottery (across all lotteries and subjects). The discrepancy is measured in percent of the highest outcome of the lottery. Figure 2 shows that in $35 \%$ of all cases the subjects adjusted their prices by more than $10 \%$ of the highest outcome of the lottery. ${ }^{3}$

\footnotetext{
${ }^{2}$ Subjects knew that the pricing task was terminated after ten seconds at the earliest.

${ }^{3}$ Subjects had to choose prices from a list of prices that were spaced equally apart. The distance between prices on the list was between $3.125 \%$ and $3.57 \%$ of the highest lottery outcome. The spike in the number of price adjustments between 5 and $10 \%$ of the highest lottery outcome is caused by the spacing of prices on the list.
} 


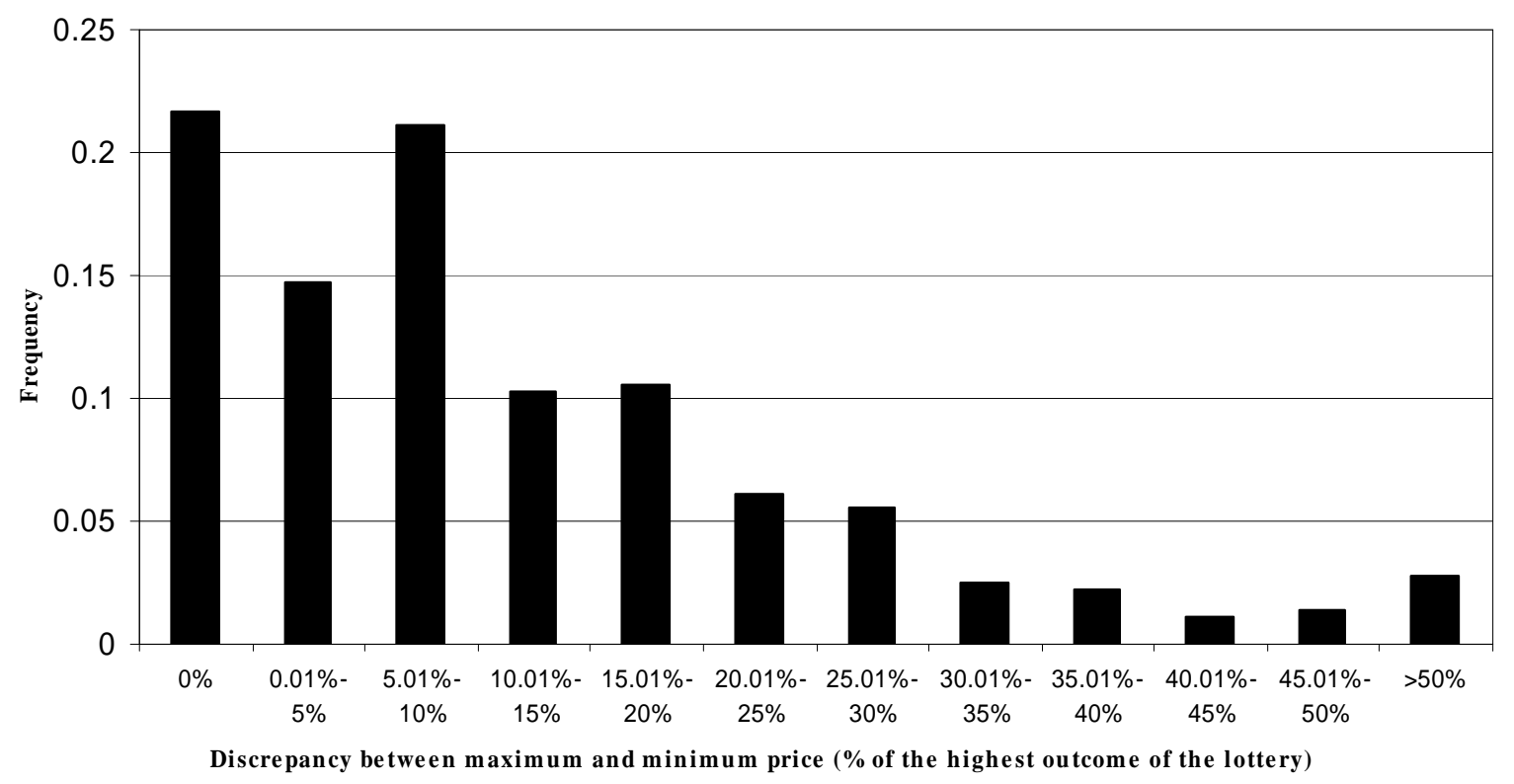

Figure 2 Discrepancy between the maximum and the minimum stated price for a lottery

Among the 360 tasks (6 lotteries and 60 subjects), there are five tasks where a subject states ten or more prices. Subject 5 stated a sequence of 13 prices for lottery 3 where all prices alternate between 33 and 36 . Subject 14 stated a sequence of 17 prices for both lottery 2 and lottery 3 . For lottery 2 , after the $4^{\text {th }}$ price, prices alternate between 20 and 24. For lottery 3, after the $2^{\text {nd }}$ price, prices alternate between 21 and 24 . Across all lotteries, subjects 5 and 14 adjusted their prices more frequently than any other subject. Subject 44 stated 10 prices for lottery 2 where the last 8 prices alternate between 28 and 32. Subject 53 stated 11 prices for lottery 2 where all prices alternate between 20 and $24 .{ }^{4}$

Next we look at the patterns of price adjustments. Let $p_{1}, p_{2}, \ldots$ be the first, second, etc., price that a subject states. We classify all pricing patterns where subjects made at least three clicks into five classes:

- "Monotone": Either $p_{t}<p_{t+1}$ for all $t$ or $p_{t}>p_{t+1}$ for all $t$.

\footnotetext{
${ }^{4}$ There is only one observation where a subject states the same price repeatedly without stating a different price in between (for lottery 1, subject 14 states the prices 45, 20, 25, 20, 20, 20). Obviously, stating a price repeatedly has no effect on payoffs. To simplify notation we treat this sequence as 45, 20, 25, 20.
} 
- "Oscillating": $p_{t}=p_{t+2}$ and $p_{t} \neq p_{t+1}$ for all $t$.

- "Fine-tuning": A non-monotone pricing pattern where the following holds: If $p_{t-1}<p_{t}$ then $p_{s}>p_{t-1}$ for all $s \geq t$. If $p_{t-1}>p_{t}$ then $p_{s}<p_{t-1}$ for all $s \geq t$.

- "Weak fine-tuning": A non-oscillating pricing pattern where the following holds: If $p_{t-1}<p_{t}$ then $p_{s} \geq p_{t-1}$ for all $s \geq t$. If $p_{t-1}>p_{t}$ then $p_{s} \leq p_{t-1}$ for all $s \geq t$. And at least one inequality holds with equality.

- "Jumping": All other pricing patterns.

Figure 3 in the Appendix gives examples for the different pricing patterns. Note that weak fine-tuning patterns can not be observed if a subject states less than four prices. Table 3 list the distribution of observed pricing patterns given the number of prices that subjects state.

\begin{tabular}{|c|c|c|c|c|c|c|}
\hline \multirow{2}{*}{$\begin{array}{l}\text { Price adjustment } \\
\text { patterns }\end{array}$} & \multicolumn{5}{|c|}{ Number of stated prices } & \multirow{2}{*}{ Total } \\
\hline & 3 & 4 & 5 & 6 & 7 or more & \\
\hline Monotone & 35 & 17 & 3 & 3 & 0 & 58 \\
\hline Oscillating & 10 & 9 & 2 & 3 & 2 & 26 \\
\hline Fine-tuning & 17 & 14 & 7 & 0 & 0 & 38 \\
\hline Weak fine-tuning & - & 16 & 10 & 6 & 1 & 33 \\
\hline Jumping & 2 & 6 & 8 & 7 & 11 & 34 \\
\hline Total & 64 & 62 & 30 & 19 & 14 & 189 \\
\hline
\end{tabular}

Table 3 The frequency of price adjustment patterns in the experiment

Table 3 shows that pricing patterns suggest that subjects search for the optimal minimum selling price. Note that it is not surprising that the frequency of monotone pricing patterns decreases if subjects state more prices and that the frequency of jumping patterns increases. Consider the first $n-1$ prices of a pattern with $n$ prices. If the first $n-1$ prices are monotone and increasing, then the complete pattern is monotone if and only if the last price is larger than all other prices. But if the first $n-1$ prices constitute a jumping pattern, then the complete pattern is jumping regardless of the last price. 


\section{Model of Random Pricing}

In our experiment, we observe that in $82 \%$ of cases, subjects state a price and later adjust the price. The vast majority of decision theories is concerned with explaining and predicting prices for lotteries but does not discuss how these prices are determined. Since we observe that subjects adjust their price, the question arises whether these price adjustments are random or whether the observed price adjustments allow to draw conclusions on how subjects determine the minimum selling price of a lottery.

In this section, we analyze whether a general class of random pricing models can explain the price patterns that we observe in the experiment. Since we observe that subjects click on several prices, we consider random pricing models that generate a sequence of minimum selling prices. Recall that in our experiment, subjects can only state prices from the list of possible prices. Hence we assume that minimum selling prices are drawn from the list of possible prices.

Definition 1: A random pricing model generates a sequence $c_{1}, c_{2}, \ldots$ of minimum selling prices where $c_{1}, c_{2}, \ldots$ are independent and identically distributed random variables.

In section 3, we classify five different pricing patterns. Without additional assumptions on the distribution of prices, random pricing models generate testable predictions only with respect to the relative probability of monotone, fine-tuning and jumping price patterns (when all prices in the sequence are different). The relative probability that a monotone sequence is observed is equal to the probability that a monotone sequence is observed divided by the probability that a monotone, fine-tuning or jumping sequence is observed. The relative probabilities of fine-tuning and jumping sequences are defined in the same way. 
Proposition 1. Consider a sequence of $n \geq 2$ different prices. If the sequence is generated by a random pricing model, then the relative probability of monotone sequences is $2 / n !$, the relative probability of fine-tuning sequences is $\left(2^{n-1}-2\right) / n !$, and the relative probability of jumping sequences is $1-2^{n-1} / n$ !.

The proof is in the Appendix.

Table 4 shows the frequency of monotone, fine-tuning and jumping pricing patterns where all prices are different. Column 2-4 lists the frequency of pricing patterns for all pricing patterns that consist of exactly three, four, and five prices. ${ }^{5}$ Among pricing patterns with seven or more prices, we do not observe monotone, fine-tuning or jumping pricing patterns where all prices are different. The probabilities that are predicted by Proposition 1 are in parentheses. The null-hypothesis is that probabilities are generated by a random pricing model as described by Proposition 1. Test statistics for pricing patterns with three or four prices refer to Pearson's Chi-squared test. Since there are only few observations for pricing patterns with five or six prices, the p-value is computed by a multinomial test.

\begin{tabular}{|c|c|c|c|c|}
\hline \multirow{2}{*}{$\begin{array}{c}\text { Price adjustment } \\
\text { pattern }\end{array}$} & \multicolumn{4}{|c|}{ Number of prices in a sequence (n) } \\
\cline { 2 - 5 } & $\mathbf{3}$ & $\mathbf{4}$ & $\mathbf{5}$ & $\mathbf{6}$ \\
\hline Monotone & $35(0.33)$ & $17(0.08)$ & $3(0.02)$ & $3(0.003)$ \\
\hline Fine-tuning & $17(0.33)$ & $14(0.25)$ & $7(0.12)$ & $0(0.042)$ \\
\hline Jumping & $2(0.33)$ & $4(0.67)$ & $2(0.87)$ & $0(0.956)$ \\
\hline$\chi^{2}$-test $(\boldsymbol{p}$-value) & 30.33 & 87.17 & $(1.07 * \mathrm{e}-08)$ & $(2.14 * \mathrm{e}-8)$ \\
\hline
\end{tabular}

Table 4 Actual frequency (predicted probability) of monotone, fine-tuning and jumping price adjustment patterns where all prices are different.

\footnotetext{
${ }^{5}$ If there are only two prices, then either both prices are the same or the sequence is monotone. Hence, the shortest price sequence where it is possible to observe monotone as well as fine-tuning and jumping patterns consists of three prices.
} 
The prediction of Proposition 1 is strongly rejected by our experimental data for sequences with six or less prices, as shown in Table 4. Hence, random pricing models can not explain the price patterns that we observe in the experiment. Intuitively, random pricing models predict that future price adjustments do not depend on the past history but we observe in the experiment that earlier prices contain information about future price adjustments.

\section{A Model of Stochastic Pricing}

Blavatskyy and Köhler (2009) propose the following model of stochastic pricing. Each individual is characterized by a finite set $\Pi$ of rational preference relations on the space of lotteries $\mathscr{L}$ and a probability measure $\eta$ that is defined on the power set of $\Pi$. We denote the elements of $\Pi$ by $\succsim_{\rho} \in \Pi$. Preferences are described by a pair $(\eta, \Pi)$. If an individual faces a binary choice problem, she draws a preference relation $\succsim_{\rho} \in \Pi$ with probability $\eta\left(\left\{\succsim_{\rho}\right\}\right)$ and chooses according to the realized $\succsim_{\rho}$.

To determine the minimum selling price of a lottery, the individual uses a sequence of binary comparisons where the lottery is compared to different monetary amounts. The sequence of comparisons starts with some amount that is drawn at random from the set of possible prices. If the lottery is preferred over an amount, then the individual concludes that the minimum selling price should be higher. Hence the amount is increased by $\Delta$ and a new comparison is made. Similar, if the amount is preferred over the lottery, then the amount is decreased by $\Delta$ and a new comparison is made. This model of stochastic pricing does not only predict the minimum selling price that individuals state for a lottery but it also describes the procedure how individuals determine the minimum 
selling price. Thus, this model has the potential for explaining the patterns of price adjustments that we observed in our experiment.

In our experiment, subjects have to select a minimum selling price from a list of equally spaced prices. Hence the set of possible prices $S_{L}$ consists of all prices on the list. ${ }^{6}$ Since subjects have to select a minimum selling price from $S_{L}$, there is no need for comparing the lottery to some price that is not in $S_{L}$. Thus, the step size $\Delta>0$ by which the amount is adjusted has to be a multiple of the distance between prices in $S_{L}$.

Let the subscript $t$ denote the number of the comparison. If an individual is asked to state the minimum selling price $P_{L} \in S_{L}$ for a lottery $L \in \mathscr{L}$, then she uses the following procedure $P$ :

1) Draw an amount $x \in S_{L}$ at random.

2) With probability $\gamma_{t} \in(0,1]$ the sequence of comparisons stops and $P_{L}=x$. Otherwise, draw a preference relation $\succsim_{\rho} \in \Pi$ according to probability measure $\eta$.

3) If $x \sim \rho L$, then $P_{L}=x$. If $x>_{\rho} L$ (if $L>_{\rho} x$ ) then step 2 is repeated where $x$ is replaced by $\max \left\{x-\Delta, \min S_{L}\right\}$ (by $\min \left\{x+\Delta, \max S_{L}\right\}$ ).

The idea behind the procedure is that individuals use a sequence of binary comparisons to determine the minimum selling price and that outcomes of earlier comparisons matter in the sense that they determine which amount is compared to the lottery in the next comparison.

In the experiment, subjects have an incentive to state the best estimate of their minimum selling price at every point in time, because the pricing task may be terminated at any point in time. Therefore, we assume that individuals state the amount that is currently

\footnotetext{
${ }^{6}$ Wilcox (1994, p.318) provides evidence that subjects search between the highest and the lowest outcome of a lottery to find their minimum selling price
} 
compared to the lottery with probability $\theta$ as price even if they are not indifferent between the amount and the lottery and the sequence of comparisons continues. Since it takes time to state a price, we expect that individuals do not state every amount that is compared to the lottery, i.e. that $\theta<1$.

We run a Monte-Carlo simulation to analyze the predictions of the model. The set of possible prices $S_{L}$ is $\{0,1, \ldots, \mathrm{N}-1\}$, where $\mathrm{N}$ is the number of prices on a grid for a corresponding lottery (cf. Table 1). The preferences of a representative individual are described by a normal distribution with mean equal to the expected value of a lottery (divided by a number of points on the price grid) and a standard deviation equal to 3 grid points. If the individual prefers the amount (the lottery) then she decreases (increases) the amount by one before she makes the next comparison. We assume that after every comparison, the individual states the price that has been compared to the lottery with probability 0.1 , i.e. $\theta=0.1$. The parameters for $\gamma_{t}$ are chosen to match the observed frequency of pricing patterns of different lengths (in particular $\gamma_{1}=0.2$ and then it gradually increases). The values for $x_{1}$ are drawn from a uniform distribution over the set of possible prices $S_{L}$. For each of six lotteries used in the experiment we ran 600 Monte-Carlo simulations (recall that we used one representative subject for 60 experimental subjects).

\begin{tabular}{|c|c|c|c|c|c|c|}
\hline \multirow{2}{*}{$\begin{array}{l}\text { Price adjustment } \\
\text { patterns }\end{array}$} & \multicolumn{5}{|c|}{ Number of stated prices } & \multirow{2}{*}{ Total } \\
\hline & 3 & 4 & 5 & 6 & 7 or more & \\
\hline Monotone & $35(375)$ & 17 (198) & $3(31)$ & $3(3)$ & $0(1)$ & $58(608)$ \\
\hline Oscillating & $10(113)$ & $9(24)$ & $2(3)$ & $3(0)$ & $2(0)$ & $26(140)$ \\
\hline Fine-tuning & 17 (147) & $14(112)$ & $7(37)$ & $0(13)$ & $0(1)$ & 38 (310) \\
\hline Weak fine-tuning & - & $16(124)$ & $10(64)$ & $6(26)$ & $1(9)$ & 33 (223) \\
\hline Jumping & $2(75)$ & $6(265)$ & $8(225)$ & $7(145)$ & $11(164)$ & 34 (874) \\
\hline Total & $64(710)$ & $62(723)$ & $30(360)$ & 19 (187) & $14(175)$ & 189 (2155) \\
\hline
\end{tabular}

Table 5 The frequency of price adjustment patterns in the experiment and in a Monte Carlo simulation of the model of stochastic pricing (in parentheses) 
Table 5 compares the actual frequency of price adjustment patterns that we observed in the experiment and the corresponding frequency from a Monte Carlo simulation of the model of stochastic pricing. Table 5 clearly shows that the model of stochastic pricing matches the qualitative properties of price adjustment patterns that we observed in the experiment. In particular, we observe in a simulation that the frequency of monotone, oscillating, fine-tuning and weak fine-tuning price adjustment patterns decreases with the number of stated prices. The simulation also shows that while monotone pricing patterns are modal patterns in relatively short sequences (3-4 prices), jumping pricing patterns become modal patterns in relatively long sequences ( 5 and more prices). Thus, we can conclude that the model of stochastic pricing accommodates the empirical findings from our experiment.

\section{Conclusion}

This paper presents the results of a new experiment where subjects state their minimum selling prices for risky lotteries under time pressure. The novel feature of our experimental design is that we record price adjustments that subjects make before they settle on a final price (or the time for decision runs out). We observe that subjects make frequent and significant price adjustments within a short period of time. We classify all observed patterns of price adjustment into five categories: monotone, oscillating, finetuning, weak fine-tuning and jumping. We show that random pricing models cannot explain the observed frequencies of these pricing patterns i.e. subjects do not make their adjustments at random. We also show that a model of stochastic pricing, where subjects search for their minimum selling price via a sequence of binary comparisons, qualitatively matches the experimental results. Thus, our experimental results suggest that people use simple procedural models to find their minimum selling price. 


\section{References}

Becker, Gordon M., Morris H. DeGroot, and Jacob Marschak (1964). "Measuring utility by a single-response sequential method" Behavioral Science 9, 226-232

Ben Zur, H., \& Breznitz, S. J. (1981). The effect of time pressure on risky choice behavior. Acta Psychologica, 47, 89-104

Blavatskyy, Pavlo, and Wolfgang Köhler (2009) "Range Effects and Lottery Pricing” Experimental Economics forthcoming

Busemeyer, J. R. (1985). Decision making under uncertainty: A comparison of simple scalability, fixed sample, and sequential sampling models. Journal of Experimental Psychology, 11, 538-564

Busemeyer, J. R. (1993) Violations of the speed-accuracy trade-off relation: decreases in decision accuracy with increases in decision time. In: O. Svenson and A.J. Maule, Editors, Time Pressure and Stress in Human Judgment and Decision Making, Plenum, New York (1993), pp. 181-193.

Fischbacher, Urs (2007) “z-Tree: Zurich Toolbox for Ready-made Economic Experiments” Experimental Economics 10(2), 171-178

Johnson, Joseph G. and Jerome R. Busemeyer (2005) “A dynamic, stochastic, computational model of preference reversal phenomena" Psychological Review, 112, 841-861.

Tversky, Amos and Daniel Kahneman (1992) “Advances in prospect theory: Cumulative representation of uncertainty" Journal of Risk and Uncertainty 5, 297-323

Wilcox, Nathaniel (1994) “On a Lottery Pricing Anomaly: Time Tells the Tale” Journal of Risk and Uncertainty 8, 311-324

Wright, P. (1974). The harassed decision maker: Time pressures, distractions, and the use of evidence. Journal of Applied Psychology, 59, 555-561 


\section{Appendix}

\section{Proof of Proposition 1}

Let $\left\{p_{1}, \ldots, p_{n}\right\}$ be a vector of $n$ distinct prices. Without loss of generality, assume that prices $p_{1}, \ldots, p_{n}$ are numbered in ascending order. Let $\left\{a_{i}\right\}_{i \in\{1, \ldots, n\}}$ denote a sequence of $n$ prices that contains each of the prices $p_{1}, \ldots, p_{n}$. Let $A_{n}$ be the set of all such sequences. The set $A_{n}$ has $n$ ! elements (the number of all possible combinations of prices $p_{1}, \ldots, p_{n}$ ). Only two sequences in the set $A_{n}$ are monotone: the ascending sequence $a_{1}<\ldots<a_{n}$ and the descending sequence $a_{1}>\ldots>a_{n}$. Hence, the probability of observing a monotone sequence in the set $A_{n}$ is $2 / n !$.

A necessary condition for a fine-tuning sequence $\left\{a_{i}\right\}_{i \in\{1, \ldots, n\}}$ is that either $a_{1}=p_{1}$ or $a_{1}=p_{n}$. Let $P^{F}(n)$ be the probability that $\left\{a_{i}\right\}$ is a fine-tuning sequence. Then $P^{F}(n)$ is equal to the probability that $a_{1}=p_{1}$ and that the sequence $\left\{a_{i}\right\}_{i \in\{2, \ldots, n\}}$ is either fine-tuning or descending plus the probability that $a_{1}=p_{n}$ and that the sequence $\left\{a_{i}\right\}_{i \in\{2, \ldots, n\}}$ is either finetuning or ascending. Therefore, $P^{F}(n)$ can be written recursively as:

$$
P^{F}(n)=(2 / n)\left(P^{F}(n-1)+1 /(n-1) !\right) .
$$

Since $P^{F}(2)=0$, we can rewrite equation (A1) as

$$
P^{F}(n)=2 / n !+4 / n !+\ldots+2^{n-2} / n !=\left(2^{n-1}-2\right) / n !
$$

If neither $a_{1}=p_{1}$ nor $a_{1}=p_{n}$ it must be the case that the sequence $\left\{a_{i}\right\}_{i \in\{1, \ldots, n\}}$ is jumping. Let $P^{J}(n)$ be the probability of observing a jumping sequence in the set $A_{n}$. Then $P^{J}(n)$ can be written recursively as:

$$
P^{J}(n)=(n-2) / n+(2 / n) P^{J}(n-1) .
$$

Since $P^{J}(2)=0$, we can rewrite the recursive equation (A2) as $P^{J}(n)=1-2^{n-1} / n$ !. Q.E.D. 
Figure 3 Examples for pricing patterns (all pricing patterns are for lottery 2)

Monotone Pricing Pattern (Subject 56, Lottery 2)

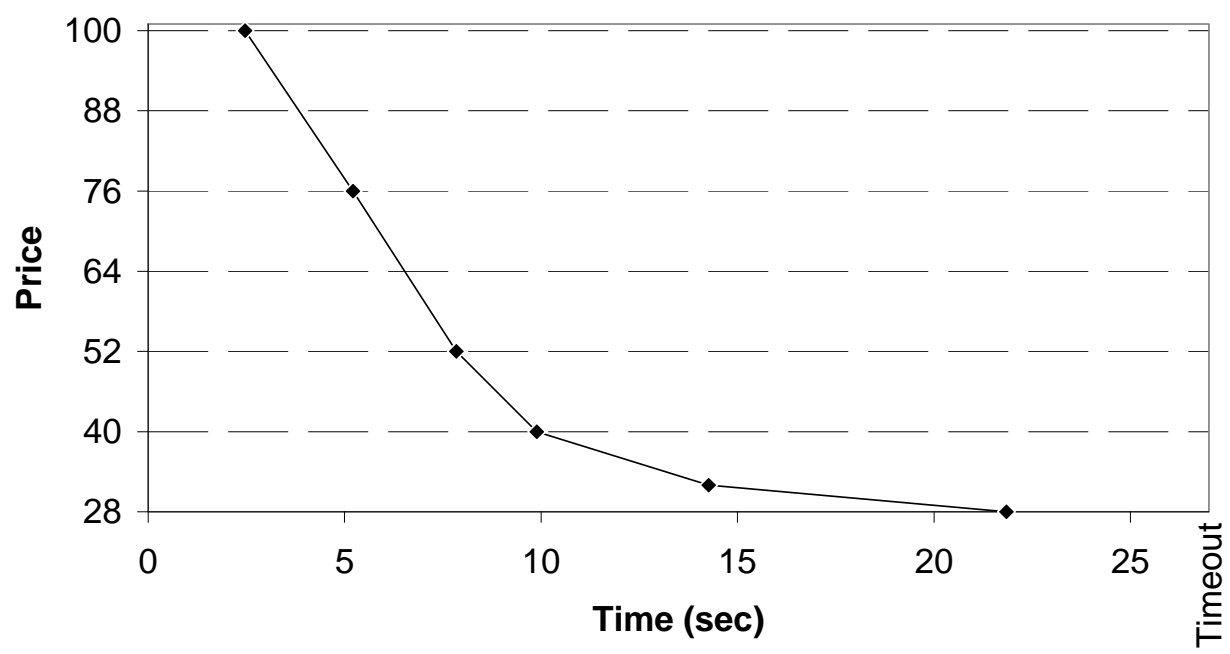

Oscillating Pricing Pattern (Subject 53, Lottery 2)

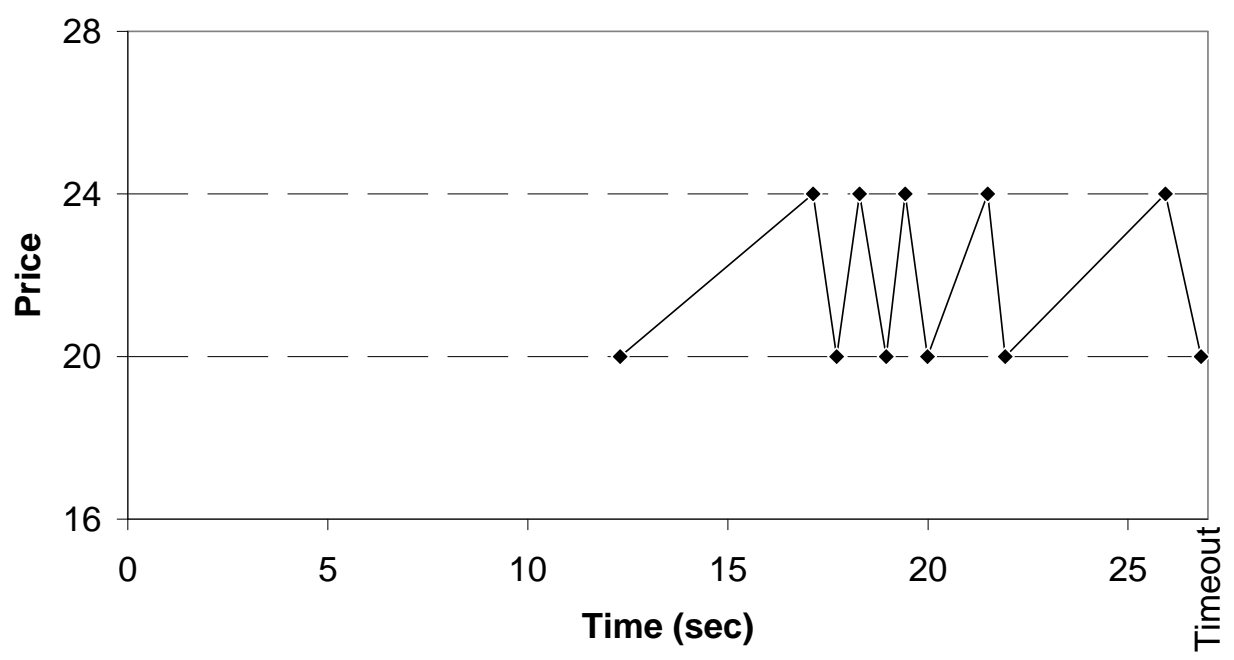

Fine-Tuning Pricing Pattern (Subject 51, Lottery 2)

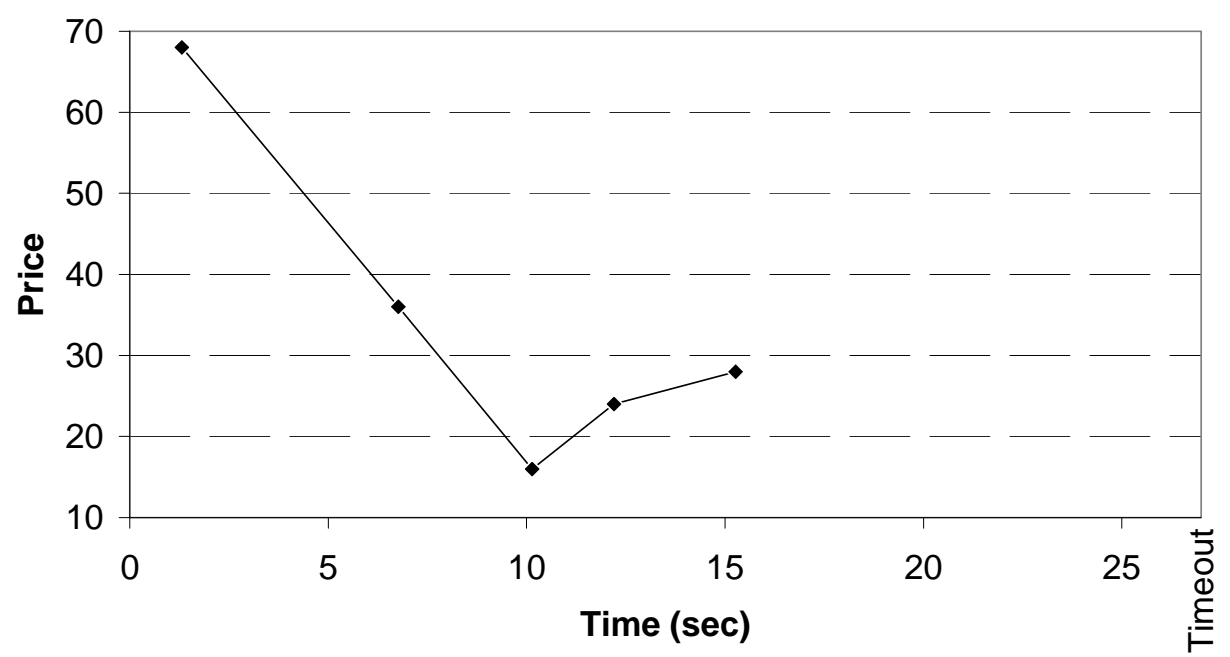


Weak Fine-Tuning Pricing Pattern (Subject 44, Lottery 2)

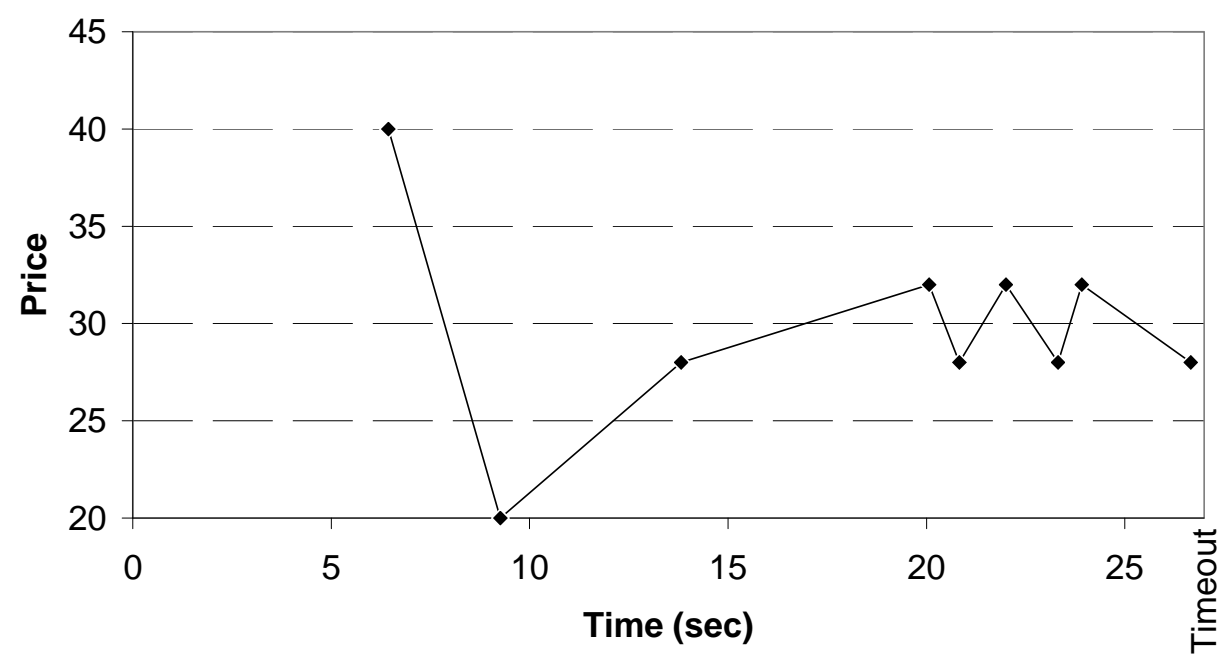

Jumping Pricing Pattern (Subject 32, Lottery 2)

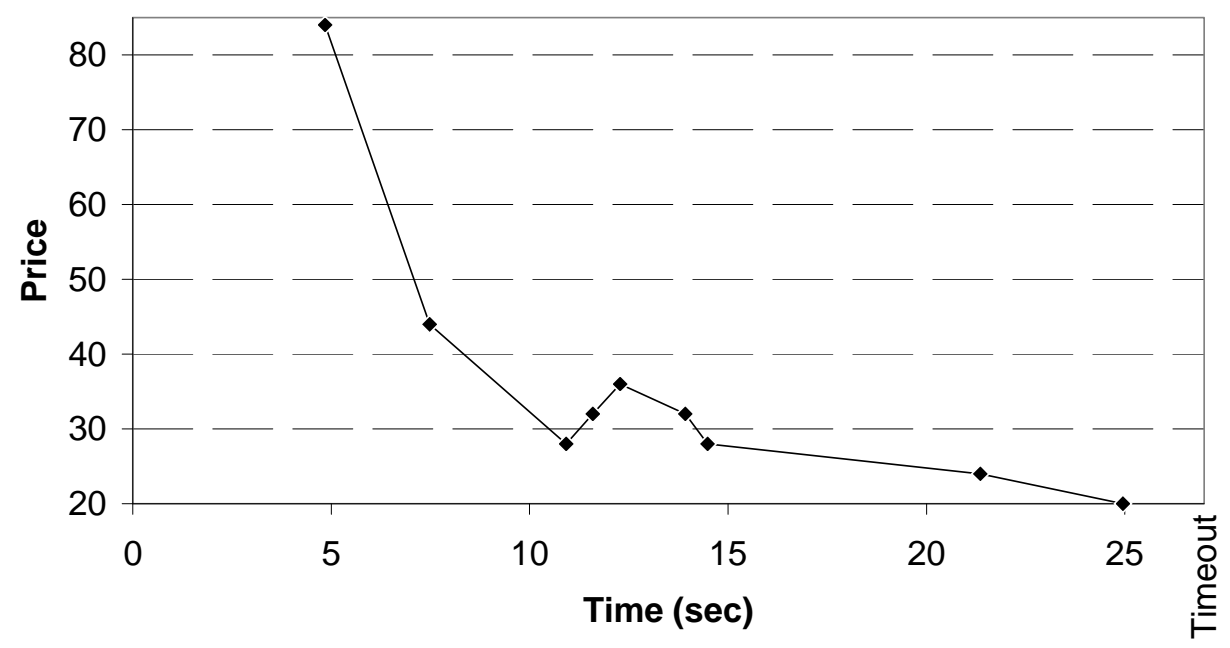




\section{Translation of the instructions for the experiment. Text in italics did not appear in the instructions.}

\section{Instructions for the Experiment}

Thank you for participating in this experiment.

This is an experiment in decision-making. The money to conduct this experiment has been provided by a research grant. We will ask you to answer 72 questions.

At the end of the experiment, we will compute how much money you receive.

Your payoff depends only on your decisions and on chance events. Your payoff does not depend on decisions of other participants.

The experiment uses different lotteries. If you play a lottery, you receive a certain amount of money with some probability. With the remaining probability, you receive nothing. All lotteries have the following structure:

A box contains 100 cards.

28 cards are red.

72 cards are black.

If a red card is drawn, you earn 110 Swiss Francs.

If a black card is drawn, you earn nothing.

At the end of the experiment appears a message on your screen that asks you to raise your hand to inform the experimenters that you have answered all questions. One of the experimenters will come by and ask you to complete a short questionnaire. Additionally, you will draw a number that determines which question is used to compute your payoff.

Depending on how you answered this question, you will either receive a fixed amount or you will play a lottery. The actual payout happens in the room in front of the computer lab. If you play a lottery, you will be asked there to draw a card from the corresponding box. The color of the card determines whether you have won in the lottery.

There is no such thing as a right or wrong answer.

There are 5 different types of questions. We use colors to distinguish the different types of questions. Colors have no meaning except to distinguish the different types of questions. 


\section{Pink}

In a pink question you own the right to play a lottery and receive the outcome. However, you can sell the lottery. In these questions, we will ask you to choose the minimum price at which you are willing to sell the right to play the lottery from a list of prices.

There are three differences compared to the lilac and green questions:

(lilac and green questions are lottery pricing tasks where payoffs are determined by the Becker-DeGroot-Marschak procedure)

1) You can not enter any price you want. Instead, you have to choose a price from the list of prices.

2) You should choose a price quickly because otherwise it is possible that your payoff is zero.

3) You can change the price as often as you want.

On the computer screen, a pink question looks like this:

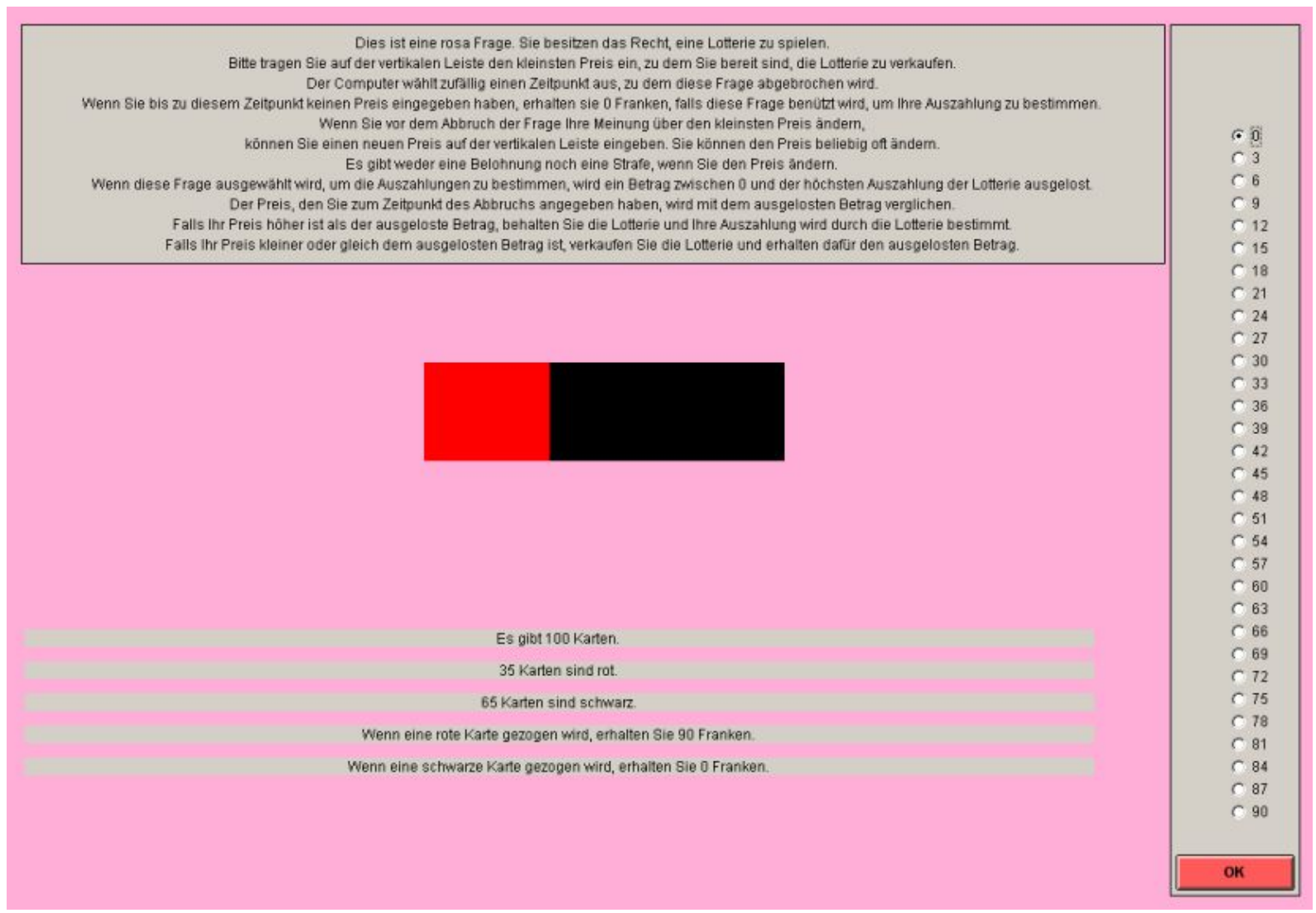

Pink Questions are terminated after a randomly determined time (at the earliest after 10 seconds, and not later than 30 seconds). If you did not click a price before the question is terminated, you will receive 0 Swiss Francs if this question is selected to determine your payoffs. 
If you change your opinion about the minimum selling price before the question is terminated, you can click a new price from the list. You can change the price as often as you want. There is no penalty or reward if you change the price.

If a pink question is selected to determine your payoffs, we will additionally draw a random amount between zero and the highest outcome of the lottery. The random amount will be compared with the last price that you entered before the question is terminated. If your price is higher than the amount that we have drawn, you will keep the lottery and your payoff is determined when you play the lottery. If your price is lower or equal to the amount that we have drawn, you will sell the lottery and receive the amount that we have drawn.

At this point, the instructions contained a reference to explanation of a lottery pricing task under the standard Becker-DeGroot-Marschak procedure that was described earlier:

Since your payoff is determined with the same method as in the lilac questions, it is also optimal in pink questions to enter the minimum price at which you are willing to sell the lottery.

For the lilac questions, the explanation why it is optimal to state the minimum price at which you are willing to sell the lottery is as follows:

\section{Question: Is it indeed optimal for you to state truthfully the minimum price at which you are willing to sell the lottery?}

\section{Answer: Yes}

\section{Why?}

The price that you enter has no effect on the amount that you receive if you sell the lottery. Your price only determines in which cases you sell the lottery. It is optimal for you to sell the lottery if you receive at least as much as the lottery is worth to you. Hence it is optimal for you to enter the minimum price at which you are willing to sell the lottery.

\section{Example:}

Suppose that you are indifferent whether you play the lottery or receive 20 Swiss Francs. If you enter a price below 20 Francs (e.g., 15 Francs), it is possible that you sell the lottery for less than 20 Francs (e.g., 17 Francs). But since the lottery is worth 20 Francs to you, you would have been better off if you would have entered a higher price and would have kept the lottery.

If you enter a price above 20 Francs (e.g., 25 Francs), it is possible that an amount between 20 and 25 Francs is randomly drawn (e.g., 23 Francs). In this case, you would keep the lottery. But since the lottery is worth 20 Francs to you, you would have been better off if you would have entered a lower price and would have sold the lottery.

Hence: It is optimal for you to enter the minimum price at which you are willing to sell the lottery. 